\title{
DPSK Signal Regeneration with a Dual-Pump Non-Degenerate Phase-Sensitive Amplifier
}

\author{
Paola Frascella, Student Member, IEEE, Stylianos Sygletos, Fatima C. Garcia Gunning, Member, \\ IEEE, Ruwan Weerasuriya, Lars Grüner-Nielsen, Richard Phelan, James O’Gorman, Andrew D. Ellis
}

\begin{abstract}
We demonstrate, for the first time to our knowledge, regeneration of a $42.66 \mathrm{Gbit} / \mathrm{s}$ differential phase shift keyed signal using a dual-pump non-degenerate four wave mixing-based fiber optic parametric amplifier. The regenerative performance of the subsystem is characterized in terms of biterror-rate against narrow-band and wide-band introduced noise. Whilst a strong receiver sensitivity improvement, up to $20 \mathrm{~dB}$, is noticed against narrow-band noise, against quasi-random (wideband) noise we observe a regeneration of $2.7 \mathrm{~dB}$.
\end{abstract}

Index Terms - differential phase shift keying (DPSK), optical parametric amplification, phase-sensitive amplification (PSA)

\section{INTRODUCTION}

A LL-OPTICAL regenerators are expected to extend the transparent length of high-speed transmission systems and simultaneously lower the energy consumption, compared to their opto-electro-optical counterparts [1]. The emergence of differential phase shift keying (DPSK) as a widely adopted modulation format for long haul transmission has prompted great interest in regeneration schemes for phase encoded signal formats. Such regenerators should provide suppression of the accumulated noise in both the amplitude and the phase of the propagated signal.

Phase sensitive amplification (PSA) has proved its potential for phase noise suppression on DPSK signals. Initial schemes were based on single pump degenerate four wave mixing (FWM) in a fiber [2], [3]. Regeneration based on dual pump degenerate fiber optic parametric amplifier (FOPA) has also been proved both theoretically [4] and experimentally [5]. Using the phase locking scheme proposed in [6] to generate a pair of synchronized and frequency symmetric pump signals, the first practical "black-box" regenerator has been demonstrated in [7]. The regenerative properties of those

Manuscript received Dec 9, 2010. This material is based upon work supported by Science Foundation Ireland under Grant 06/IN/I969 and by the European Communities Seventh Framework Programme FP/2007-2013 under grant 224547 (PHASORS)

P. Frascella, S. Sygletos, F. C. Garcia Gunning, R. Weerasuriya, and A. D. Ellis are with Photonic Systems Group, Tyndall National Institute, Lee Maltings, Prospect Row, and Department of Physics, University College Cork, Cork, Ireland (e-mail: \{paola.frascella, stylianos.sygletos\}@tyndall.ie ).

L. Grüner-Nielsen is with OFS, Priorparken 680, 2605 Brøndby, Denmark.

R. Phelan is with Eblana Photonics, Trinity College Enterprise Centre, Pearse Street, Dublin 2, Ireland.

J. O'Gorman is with Xylophone Optics Ltd, 28 Orchardstown Drive, Rathfarnham, Dublin 14, Ireland.

Copyright (C) 2011 IEEE schemes have been assessed by applying a periodic (single tone) degradation to their inputs.

PSA is also feasible with a dual pump non-degenerate FOPA scheme [8]. This is particularly attractive for wavelength division multiplexing (WDM) applications because it can provide broadband amplification and polarization independent performance and has lower pump power consumption than single pump FOPA [9]. However, although the amplification properties of the dual-pump nondegenerate scheme have been extensively studied [10], its regenerative properties have not been explored.

In this letter, we describe the regeneration of $42.66 \mathrm{Gbit} / \mathrm{s}$ DPSK signals using a non-degenerate dual-pump FWM based phase-sensitive FOPA. The idler for the PSA and the pilot tones for pump phase-locking were derived from a frequency comb. We have assessed, for the first time to the best of our knowledge, the performance of a non-degenerate DPSK regenerator in terms of receiver sensitivities under different phase noise conditions. Complete regeneration of periodic distortion (narrow bandwidth noise) was achieved and up to $2.7 \mathrm{~dB}$ regeneration of quasi random phase noise (bandwidth exceeding the signal bandwidth) was observed.

\section{EXPERIMENTAL Details}

The experimental setup is illustrated in Fig. 1. We generated a phase coherent multi-tone signal using a comb generator based on two Mach-Zehnder modulators $(\sim 10 \mathrm{~dB}$ insertion loss) driven by a $42.66 \mathrm{GHz}$ sine wave. The comb flatness was maintained by a feedback loop, which monitored the spectrum with a Fabry-Perot filter and adjusted the modulators' bias. A tap from the comb signal was used to generate two pilot carriers spaced by $426.6 \mathrm{GHz}$, whilst the remaining signal was filtered to obtain a signal-idler pair (two subcarriers spaced by $85.32 \mathrm{GHz}$ ). The latter were data encoded to produce two $42.66 \mathrm{Gbit} / \mathrm{s}$ DPSK WDM channels, carrying a pseudo random binary sequence (PRBS) with a $2^{31}$ - 1 pattern length. Phase noise was added by driving a phase modulator with a $56 \mathrm{Gbit} / \mathrm{s}$ pattern generator operated from an independent clock. We added two different kind of phase noise: 1) a periodic degradation, by programming the pattern generator with a " 1100 " pattern giving a $14 \mathrm{GHz}$ square wave distorting signal; 2) a quasi-random distortion, in this case a $56 \mathrm{Gbit} / \mathrm{s} 2^{15}-1$ PRBS pattern was selected. In the latter case, the resultant phase noise spectrum exceeded the optical bandwidth of the receiver, and consequently it could be 


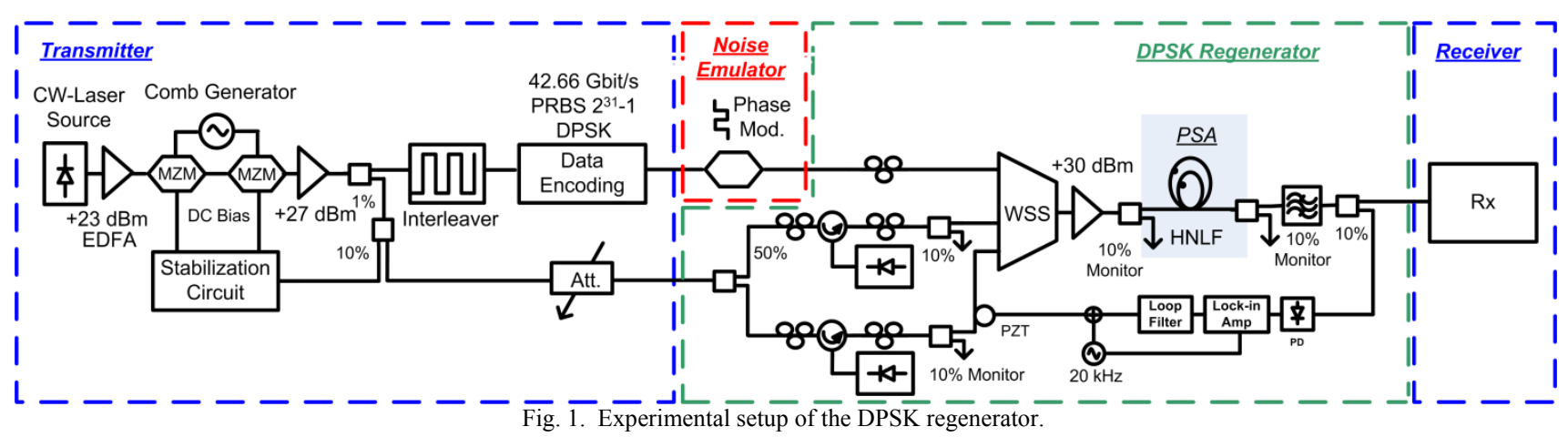

considered as a quasi-random phase degradation. The optical signal-to-noise ratio at the input of the PSA, (total signal and idler power divided by noise power in $0.1 \mathrm{~nm}$ ) was $44 \mathrm{~dB}$.

At the DPSK regenerator input, the pilot carriers with powers of $\sim-17 \mathrm{dBm}$ were used to phase and frequency synchronize two discrete-mode laser [6] based CW pumps by injection locking. This process locally regenerates the seeds by removing their amplitude noise and by amplifying them. The pumps were then combined with the signal and idler using a commercially available continuous band wavelength selective switch (WSS). The combined signals were then amplified to a total power of $30 \mathrm{dBm}$ (the two pumps contributed $93 \%$ of the total power) at the input of PSA and launched into a $210 \mathrm{~m}$ length of highly-nonlinear fiber (HNLF), which had $14 \mathrm{~dB} / \mathrm{km}$ attenuation, $\quad 0.09 \mathrm{ps} / \mathrm{Jkm}$ polarization mode dispersion coefficient, $-0.20 \mathrm{ps} /(\mathrm{nm} \cdot \mathrm{km})$ chromatic dispersion and a nonlinear coefficient of $7.4(\mathrm{~W} \cdot \mathrm{km})^{-1}$ (all parameters @ $1550 \mathrm{~nm}$ ). The HNLF had an increased threshold for stimulated Brillouin scattering (SBS) of $0.8 \mathrm{~W}$, thanks to an Al-doped core [11] and applied strain. A traditional phase locked loop maximized the total output power by controlling a piezoelectric-based fiber stretcher (PZT) in one of the pump paths to compensate for any slow phase drift between the interacting waves arising from acoustic or thermal effects.

The performance of the phase distorted input and the regenerated signals were assessed using a pre-amplified receiver which employed an $85 \mathrm{GHz}$ free spectral range (FSR) asymmetric Mach-Zehnder interferometer (AMZI) and a $0.64 \mathrm{~nm}$ filter. A further $42.6 \mathrm{GHz}$ FSR AMZI was used to demodulate the DPSK signal prior to single ended detection (constructive port) and bit-error rate (BER) measurements.

\section{RESUlts AND DisCUSSIONS}

Fig. 2 illustrates the measured spectra at the output of the PSA regenerator with and without degradation induced by the periodic "1100" pattern. A gain contrast in excess of $12 \mathrm{~dB}$ was achieved for both channels simultaneously (signal and idler are equivalent or could be equivalently swapped) in absence of degradation, which was reduced when the signal was degraded by phase modulation.

The regenerative properties of the non-degenerate phasesensitive FOPA in the presence of periodic degradation are shown in Fig. 3 (a), where the BER is plotted against total received power (both channels). The regenerated BER at the output of PSA (blue) fell $1 \mathrm{~dB}$ from the BER at PSA input without degradation (black) when an input degradation of $\pm 40 \mathrm{deg}$ was introduced (red BER curves). The PSA clearly improved the BER performance and restored the eye diagrams of the degraded signals after differential detection (Fig. 3 (b)).

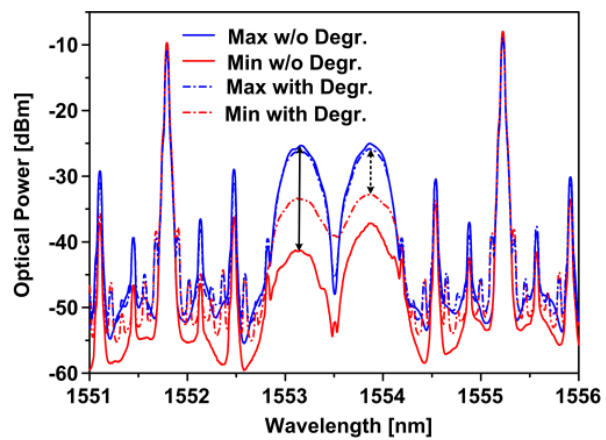

Fig. 2. Measured output spectra of PSA for non-degraded (solid line) and degraded (dashed) signals at minimum (red) and maximum (blue) gain.
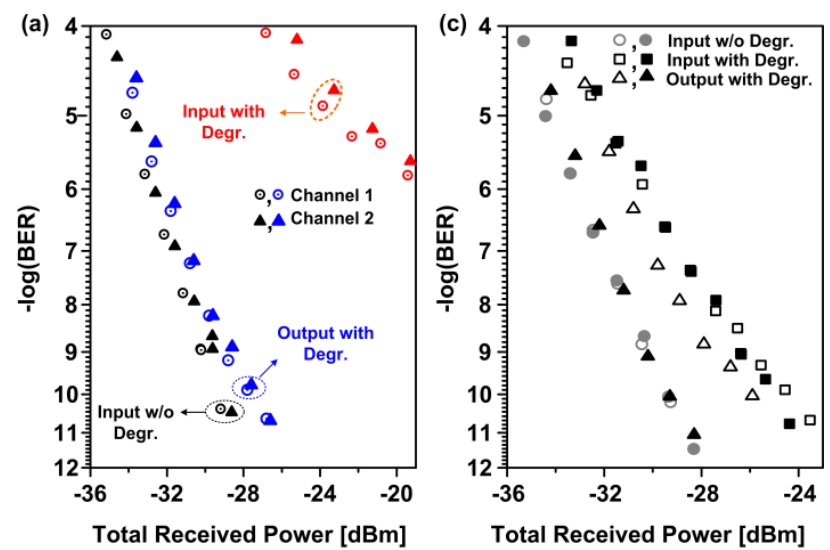

(b)

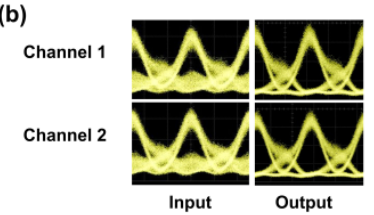

(d)

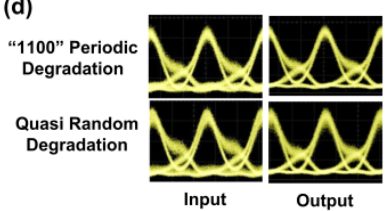

Fig. 3. (a) Measured BER characteristics and (b) eye diagrams at the input and output of the PSA regenerator in the presence of $\pm 40 \mathrm{deg}$ of periodic degradation for both channels. (c-d) Comparison of regeneration for equal initial penalties from periodic (filled symbols) and quasi-random (hollow) degradation at the regenerator input (squares) and output (triangles).

Fig. 3 (c-d) compares the performance for levels of periodic and quasi-random distortion that both give a receiver 


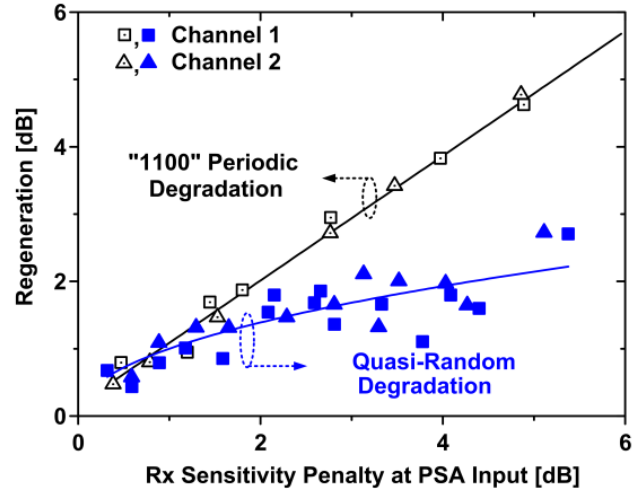

Fig. 4. Non-degenerate PSA regeneration as a function of receiver sensitivity penalty at the input of the PSA for periodic (open symbols) and quasi-random degradation (filled) for channel 1 (squares) and 2 (triangles). Solid lines are shown to guide the eye.

sensitivity penalty of $\sim 4.3 \mathrm{~dB}$ at the input of the PSA. Whereas we observed almost ideal regeneration in the case of periodic degradation, in the case of quasi-random degradation the level of regeneration was restricted to $1.6 \mathrm{~dB}$.

The saturation of the improvement with a quasi random degradation is further illustrated in Fig. 4, where the receiver sensitivity penalty at the input of PSA was varied between 0 and $5 \mathrm{~dB}$ for both types of degradation. Fig. 4 shows that the regeneration (defined as the difference between the receiver sensitivity at the output of PSA and the receiver sensitivity at the input) followed a linear behavior for the periodic degradation. For this kind of degradation, the performance of the regenerated signal was within $1 \mathrm{~dB}$ of the non-degraded back-to-back signal, even for periodic degradations leading to receiver sensitivity penalties of $20 \mathrm{~dB}$ (Fig. 3 (a)). On the other hand, for a quasi random degradation, the regeneration tended to saturate as the degradation increased, with a maximum of $2.7 \mathrm{~dB}$. Beyond the range illustrated in Fig. 3, for a quasi random degradation an error floor developed in the regenerated signal. We believe that this is because, in the presence of random phase noise, it is impossible for an optical regenerator to improve the BER of a signal beyond the limit obtained by using an ideal receiver. In this case we believe that the physical mechanism behind the limitation is the conversion of phase noise to amplitude noise. This effect is qualitatively illustrated with the constellation diagrams in Fig. 5. This figure schematically represents the degraded input signal (in both cases the added phase noise had the same standard deviation) and the corresponding output signal. For a periodic signal, whilst the gain is somewhat reduced by the phase modulation (see also Fig. 2), no significant additional amplitude noise is induced. However, for quasi-random degradation a conversion of phase noise to amplitude noise is clearly apparent, leading to a reduction in the overall signal quality. This enhanced amplitude noise at the PSA output signal for a quasi-random degradation is in stark contrast to the simple gain reduction observed for a periodic degradation. We also believe that this amplitude noise could be reduced by operating the PSA in a saturated mode, or by cascading the PSA with a phase preserving amplitude regenerator, although any associated error floors due to initial phase errors of more than 90 degrees would inevitably be locked in.

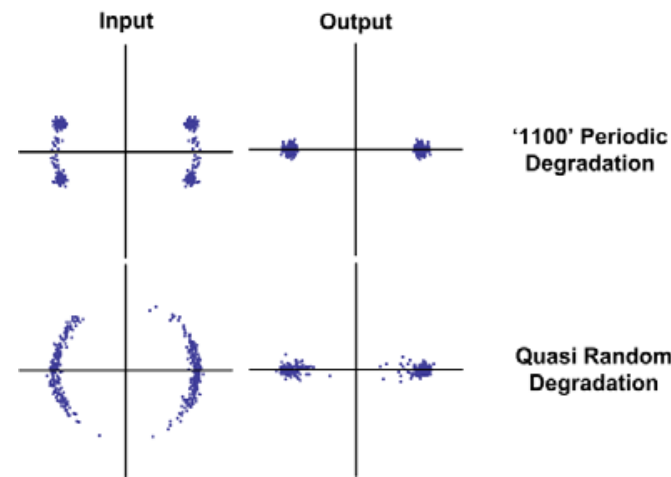

Fig. 5. Analytical prediction of normalized PSA input and output at $14 \mathrm{~dB}$ onoff gain for both degradations.

\section{CONCLUSION}

We report, for the first time, the regeneration in terms of receiver sensitivity of a $42.66 \mathrm{Gbit} / \mathrm{s}$ DPSK signal by a dual pump non-degenerate PSA. We have demonstrated that the phase transfer characteristic of the PSA provides up to $2.7 \mathrm{~dB}$ regeneration of a signal degraded by quasi random phase noise, and have identified phase to amplitude conversion as the limiting factor for regeneration performance.

\section{ACKNOWLEDGMENTS}

The authors acknowledge F. Brandonisio, S.K. Ibrahim, R.P. Webb, A. Bogris, and F. Parmigiani for their assistance.

\section{REFERENCES}

[1] S. Sygletos, I Tomkos, J. Leuthold, "Technological challenges on the road toward transparent networks," Journal of Optical Networking, vol. 7, pp. 321-350 (2008).

[2] M. E. Marhic and C. H. Hsia, "Optical amplification and squeezed-light generation in fibre interferometers performing degenerate four-wave mixing", Quantum Opt., vol. 3, pp. 341-358 (1991).

[3] K. Croussore, C. Kim, and G. Li, "All-optical regeneration of differential phase-shift keying signals based on phase-sensitive amplification", Opt. Lett., vol. 29, no. 20, pp. 2357-2359 (2004).

[4] A. Bogris, and D. Syvridis, "RZ-DPSK Signal Regeneration Based on Dual-Pump Phase-Sensitive Amplification in Fibers", IEEE Phot. Tech. Lett., vol. 18, no. 20, pp.2144-2146 (2006).

[5] K. Croussore and G. Li, "Phase regeneration of NRZ-DPSK signals based on symmetric-pump phase sensitive amplification," IEEE Photon. Technol. Lett., vol. 19, no. 11, pp.864-864 (2007).

[6] R. Weerasuriya et al., "Generation of frequency symmetric signals from a BPSK input for phase sensitive amplification," in Proc. Opt. Fiber Comm. Conf., San Diego, CA, Mar. 21-25, 2010, Paper OWT6.

[7] R. Slavík, et al., "All-optical phase and amplitude regenerator for nextgeneration telecommunications systems", Nature Photonics, vol. 4, pp. 690-695 (2010).

[8] F. S. Yang, M.C. Ho, M.E. Marhic, L.G. Kazovsky, "Demonstration of two-pump fiber optical parametric amplification," Electron. Lett., vol. 33, p 1812 (1997).

[9] S. Radic et al., "Continuous-wave parametric gain synthesis using nondegenerate pump four-wave mixing," IEEE Photon. Technol. Lett., vol. 14 , no. 10, pp.1406-1408 (2002).

[10] J. Kakande, F. Parmigiani, M. Ibsen, P. Petropoulos, and D. J. Richardson, "Experimental investigation of wide bandwidth single and dual pump non-degenerate phase sensitive amplifiers," in Proc. Opt. Fiber Comm. Conf., San Diego, CA, Mar. 21-25, 2010, Paper OWT3.

[11] L. Grüner-Nielsen et al., "A silica based highly nonlinear fibre with improved threshold for stimulated Brillouin scattering," in Proc. European Conf. on Opt. Comm., Torino-Italy, Sep. 19-23, 2010, Paper Tu.4.D.3. 